\title{
Cell-type deconvolution from DNA methylation in carotid atherosclerotic plaques using bioinformatics approach
}

\author{
Markov A. ${ }^{1 *}$, Zarubin A. ${ }^{1}$, Sharysh D. ${ }^{2}$, Golubenko M. ${ }^{1,3}$, Babushkina N. ${ }^{1}$, \\ Valiakhmetov N. ${ }^{1}$, Kazantsev A. ${ }^{3}$, Barbarash O. ${ }^{3}$, Puzyrev V. ${ }^{1,2}$, Nazarenko M. ${ }^{1,2,3}$ \\ ${ }^{1}$ Research Institute of Medical Genetics of Tomsk NRMC, Tomsk, Russia \\ ${ }^{2}$ Siberian State Medical University, Tomsk, Russia \\ ${ }^{3}$ Research Institute for Complex Issues of Cardiovascular Diseases, Kemerovo, Russia \\ *e-mail: anton.markov@medgenetics.ru
}

Key words: atherosclerosis, DNA methylation, DNA microarrays, cell-type deconvolution

Motivation and Aim: A range of aberrantly methylated genomic loci was identified in arteries during atherosclerosis, but the top lists of loci from different studies demonstrate a little overlap, possibly, due to diverse cellular composition [1]. We assessed the bioinformatic framework "EpiDISH" [2] to account for cell type heterogeneity in carotid atherosclerotic plaques (CAP).

Methods and Algorithms: We used DNA methylation (DNAme) data from Gene Expression Omnibus to build the EpiDISH reference for cell types constituting arterial tissues. Experimental specimens of CAP and blood leukocytes (PBL) were collected from 43 patients (aged $63 \pm 7$ years). Immunohistochemical analysis and genome-wide DNAme profiling (using Infinium MethylationEPIC BeadChip) were performed in 16 CAP. Methylation level of separate $\mathrm{CpG}$-sites was validated in 34 patient-matched CAP and PBL samples by NGS-based targeted bisulfite sequencing.

Results: Unsupervised cluster analysis of CAP methylomes (based on $775836 \mathrm{CpG}-$ sites) showed two main sample groups which could not be explained by clinical characteristics or type of atherosclerotic plaque. About $15 \%$ of $\mathrm{CpG}$-sites (including CpG-site cg06330621 within ZCCHC14 gene) were shown to be differently methylated between these groups (the difference between average methylation levels $|\Delta \beta|>0.1$, FDR-adjusted $p$-value $<0.05$ ). EpiDISH allowed to get approximate cell-type content, and the proportion of immune cells explained well the sample clustering. We showed a proportion of CD68+ macrophages corresponded with EpiDISH "monocyte" fraction (estimation error varied from 3 to $11 \%$ ). Validation assay of DNAme within ZCCHC14 locus showed distinct tissue-specific DNAme patterns in paired CAP and PBL samples. Conclusion: Genome-wide DNAme in carotid atherosclerotic plaques is strongly associated with underlying immune cell infiltration. An anticipated cell-type content in the whole specimen of the artery can be obtained from DNAme data. Acknowledgements: Supported by the RSF (17-75-10146).

\section{References}

1. Aavik E., Babu M., Ylä-Herttuala S. DNA methylation processes in atherosclerotic plaque. Atherosclerosis. 2019;281:168-179.

2. Teschendorff A.E., Breeze C.E., Zheng S.C., Beck S. A comparison of reference-based algorithms for correcting cell-type heterogeneity in epigenome-wide association studies. BMC Bioinformatics. 2017;18(1):105. 